\title{
CONSTELAÇÕES DE SINAIS CASADAS A GRUPOS NÃO-COMUTATIVOS
}

\author{
Antonio de Andrade e Silva*e Reginaldo Palazzo Jr. ${ }^{\dagger}$ \\ Departamento de Matemática - CCEN-UFPb \\ Departamento de Telemática - FEEC-UNICAMP
}

\begin{abstract}
Resumo - Neste trabalho consideramos a caracterização de constelaçōes de sinais casadas a grupos não-comutativos, estes como sendo resultantes do produto semidireto de um grupo comutativo por um grupo cíclico de ordem par. Como consequência, propomos um algoritmo para a determinação das constelações de sinais casadas a tais grupos.

Abstract - In this paper we consider signal constellations matched to noncommutative groups. These groups are characterized as the semidirect product of a commutative group by a cyclic group of order even. As a consequence, we propose an algorithm to determine the signal constellations matched to such groups.
\end{abstract}

Palavras Chaves: Constelações de sinais casadas a grupos não-comutativos, códigos de Slepian, rotulamento isométrico, casamento de sinais a grupos.

\section{INTRODUÇÃO}

Forney [2] mostrou que a maioria das classes de bons códigos de espaço de sinais são geometricamente uniformes. Uniformidade geométrica, além de ser um tipo forte de simetria, inclui propriedades desejáveis tais como: todas as regiões de Voronoi são congruentes; o espectro de distância é o mesmo independente da palavra-código; as palavras-código possuem a mesma probabilidade de erro; e o grupo gerador é isomorfo a um grupo de permutação transitivo.

Um dos problemas de pesquisa relacionado aos códigos geometricamente uniformes tem a ver com a estrutura de grupo do grupo gerador $G(S)$ pertencente ao grupo de simetrias $\Gamma(S)$, isto é, se $G(S)$ é o grupo gerador de $S$ (conjunto de sinais) e $s_{0} \in S, s_{0}$ um ponto inicial, então $S$ é a órbita de $s_{0}$ sob $G(S)$, e o mapeamento $\mu: G(S) \longrightarrow S$ definido por $\mu(g)=g\left(s_{0}\right)$, é um-a-um, onde $g \in G(S)$ e $g\left(s_{0}\right)$ denota a ação de $g$ no ponto inicial $s_{0}$. Este mapeamento induz uma estrutura de grupo em $S$ que é isomorfa ao grupo gerador $G(S)$.

Desse modo, o procedimento de determinação do mapeamento $\mu($.$) resulta no procedimento de casamento de sinais a$ grupos. Consequentemente, é uma maneira alternativa de se determinar códigos geometricamente uniformes.

O objetivo deste trabalho é estender o resultado de Loeliger

*O autor está no Departamento de Matemática, CCEN-UFPb, 58.059900, J. Pessoa, $\mathrm{Pb}$.

†O autor está no Departamento de Telemática, FEEC-UNICAMP, CP 6101, 13081-970, Campinas, SP, Brasil. Este trabalho foi financiado em parte pela Fundação de Amparo à Pesquisa do Estado de São Paulo, FAPESP. No. 95/4720-8, e tern recebido apoio financeiro do Conselho Nacional de Desenvolvimento Científico e Tecnológico, CNPq, No. 301416/85-0. email:palazzo@dt.fee.unicamp.br
[3], o casamento de conjunto de sinais a grupos abelianos cíclicos, no sentido de se estabelecer as condições em que um conjunto de sinais está casado a um grupo não-comutativo.

Este trabalho está organizado da seguinte maneira. Na Seção 2, estabelecemos os conceitos necessários para o entendimento das seções que se seguem. Na Seção 3, caracterizamos as constelações de sinais que são casadas a um grupo nãocomutativo resultante do produto semidireto de um grupo comutativo por um grupo cíclico de ordem par. Na Seção 4, são apresentados resultados relativos aos conceitos de $d$-caminho e de $d$-cadeia. Na Seção 5, apresentamos um algoritmo para a obtenção de constelações de sinais casadas a grupos, grupos estes formados pela extensão de dois outros grupos. Finalmente, na Seção 6, apresentamos as conclusões.

\section{PRELIMINARES}

Uma constelação de sinais $S$ é qualquer subconjunto discreto do $\mathbb{R}^{N}$. Os elementos de uma constelação de sinais $S$ são chamados pontos de sinais. Um código do espaço euclidiano é um subconjunto de $S^{\mathbb{I}}$, onde $\mathbb{I} \subseteq \mathbb{Z}$, e $S^{\mathbb{I}}$ denota o produto cartesiano de $S$, I vezes.

Uma isometria $\varphi$ é uma aplicação que preserva distância, isto é, $d\left(\varphi\left(s_{i}\right), \varphi\left(s_{j}\right)=d\left(s_{i}, s_{j}\right)\right.$.

Uma constelação de sinais $S$ é geometricamente uniforme [2] se dados $\mathbf{s}_{1}, \mathbf{s}_{2} \in S$ existe uma isometria $=\ldots$ $\operatorname{Isom}\left(\mathbb{R}^{N}\right)$, o conjunto de isometrias, tal que

$$
\varphi\left(\mathrm{s}_{1}\right)=\mathrm{s}_{2} \text { e } \varphi(S)=S .
$$

Se $\Gamma(S)=\left\{\varphi \in I \operatorname{som}\left(\mathbb{R}^{N}\right): \varphi(S)=S\right\}$, o conjunto de simetrias de $S$, isto é, o conjunto de todas as isometrias que deixam $S$ invariante, então $S$ é a órbita de qualquer ponto $\mathrm{s}_{0} \in S \operatorname{sob} \Gamma(S)$, isto é,

$$
S=\left\{\varphi\left(\mathbf{s}_{0}\right): \varphi \in \Gamma(S)\right\}=\bigcup_{\varphi \in \Gamma(S)}\left\{\varphi\left(\mathbf{s}_{0}\right)\right\}
$$

Note que $\Gamma(S)$ sob a operação de composição forma um grupo. Em geral, o grupo de simetrias $\Gamma(S)$ de uma constelação de sinais geometricamente uniforme é mais do que o necessário para gerar $S$. Assim, um grupo gerador $G(S)$ de $S$ é um subgrupo de $\Gamma(S)$ que é minimamente suficiente para gerar $S$ a partir de qualquer ponto $\mathrm{s}_{0} \in S$ ou, equivalentemente, o conjunto $\{\mathrm{s} \in S: \varphi(\mathrm{s})=\mathrm{s}\}$ tem cardinalidade nula, $\forall \varphi \in G(S), \varphi \neq I_{S}$, onde $I_{S}$ é o elemento identidade de $\Gamma(S)$. Note que o grupo gerador $G(S)$ de uma constelação de sinais geometricamente uniforme pode não existir [2]. Se 


\section{Antonio de Andrade e Silva, Reginaldo Palazzo Jr Constelaçōes de Sinais Casadas a Grupos Não-Comutativos}

$G(S)$ é o grupo gerador de uma constelação de sinais geometricamente uniforme $S$ e s $\mathrm{s}_{0} \in S$, então

$$
S=\bigcup_{\varphi \in G(S)}\left\{\varphi\left(\mathrm{s}_{0}\right)\right\},
$$

e o mapeamento $\mu: G(S) \longrightarrow S$ definido por $\mu(\varphi)=\varphi\left(\mathbf{s}_{0}\right)$ é bijetivo. O mapeamento $\mu$ induz uma estrutura de grupo em $S$, isto é, dados $\mathrm{s}_{1}, \mathrm{~s}_{2} \in S$, a operação $\mathrm{s}_{1} * \mathrm{~s}_{2}=$ $\mu\left(\mu^{-1}\left(\mathbf{s}_{1}\right) \mu^{-1}\left(\mathbf{s}_{2}\right)\right)$ define uma estrutura de grupo em $S$ e, neste caso, $(S, *)$ é isomorfo a $G(S)$. Assim, se o grupo $S$ não é simples, então cada subgrupo normal $S^{\prime}$ de $S$ induz em $S$ uma fatoração de $S^{\prime}$ por $S / S^{\prime}$. Isto sugere o estudo de uma constelação de sinais $S$ através de constelações de sinais $S^{\prime}$ e $S / S^{\prime}$ cada com cardinalidade menor do que a cardinalidade de $S$. Este fato será apresentado na Seção 3.

Um código de bloco cujas palavras-código possuem a mesma energia ou um código esférico $S$, é qualquer constelação finita de sinais sobre uma esfera que gera $\mathbb{R}^{N}$ como um espaço vetorial, [7]. Em particular, quando um código esférico $S$ é geometricamente uniforme dizemos que $S$ é uma constelação uniforme. Constelações uniformes foram introduzidas por Slepian, [7], sob o nome de códigos de grupo para o canal gaussiano e generalizadas por Forney para qualquer constelação de sinais, [2].

Seja $H$ um subgrupo normal de $G(S)$. Então,

$$
S_{g}=\bigcup_{\phi \in \varphi_{g} H}\left\{\phi\left(\mathbf{s}_{0}\right)\right\}=\bigcup_{\varphi \in H}\left\{\varphi_{g}\left(\varphi\left(\mathbf{s}_{0}\right)\right)\right\}, \varphi_{g} \in G(S)
$$

é a órbita de $\mathrm{s}_{0}$ sob a classe lateral $\varphi_{g} H$. Assim,

$$
S=\bigcup S_{g} .
$$

Se $S^{\prime}$ é a órbita de so sob $H$, então a partição $S / S^{\prime}$ de $S$ induzida por $H$ é chamada uma partição geometricamente uniforme.

Um grupo de rótulos $G$ para uma partição geometricamente uniforme $S / S^{\prime}$ é um grupo isomorfo ao grupo quociente $G(S) / G\left(S^{\prime}\right)$. Um rotulamento isométrico [2] da partição geometricamente uniforme $S / S^{\prime}$ é um mapeamento $\mu: G \longrightarrow S / S^{\prime}$ definido por

$$
\mu(g)=\varphi_{g}\left(S^{\prime}\right)
$$

Em [3] Loeliger generaliza, para um grupo finito, a idéia de rotulamento isométrico, definindo um mapeamento casado de um grupo sobre uma constelação de sinais e mostra que toda constelação de sinais casada a um grupo é, a menos de translaçâo, uma constelação uniforme, [3, Corolário 1], e caracteriza todas as constelações de sinais casadas a um grupo comutativo cíclico, [3, Teorema 10]. Este resultado é generalizado por Palazzo et al. [4] através de um algoritmo heurístico. Em todos estes casos, a motivação foi a introdução de alguma "linearidade" no estudo de códigos do espaço euclidiano via um grupo.

\section{CONSTELAÇÕES DE SINAIS CASADAS A GRUPOS}

Nesta seção iremos caracterizar as constelações de sinais que são casadas a um grupo não comutativo que é fatorável 94 como o produto semi-direto de um grupo comutativo por um grupo cíclico de ordem par.

Uma constelação de sinais $S$ é casada a um grupo $G$, [3], se existe um mapeamento sobrejetivo $\mu$ de $G$ em $S$ tal que

$$
d(\mu(g), \mu(h))=d\left(\mu(e), \mu\left(g^{-1} h\right)\right),
$$

para todo $g, h \in G$, onde $d(.,$.$) é a distância euclidiana qua-$ drática e $e$ é a identidade de $G$. O mapeamento $\mu$ é chamado de mapeamento casado. Quando o mapeamento $\mu$ é injetivo dizemos que $\mu^{-1}$ é um rotulamento casado, isto é, se $G$ é isomorfo a $G(S)$ então $\mu^{-1}$ é um rotulamento isométrico. Salvo menção explicita em contrário, todos os mapeamentos casados deste trabalho são rotulamentos casados. Neste caso, se $C$ é um código linear de comprimento $n$ sobre $G$, isto é, $C$ é um subgrupo de $G^{n}$, então $\mu(C)$ é um código do espaço euclidiano sobre $S$. Assim, as regiões de decisões de $\mu(C)$ são todas congruentes.

Sejam $H$ e $K$ dois grupos, com $H$ um subgrupo normal em $H \times{ }_{\theta} K$, e seja $\theta: K \longrightarrow A u t(H)$ um homomorfismo de grupo. Então o produto semidireto de $H$ por $K$ via $\theta$ é o conjunto $H \times_{\theta} K=\{(h, k): h \in H, k \in K\}$ junto com a operação binária

$$
\begin{aligned}
\left(h_{1}, k_{1}\right)\left(h_{2}, k_{2}\right) & =\left(\theta\left(k_{1}\right) h_{2}, k_{1} k_{2}\right), \\
\forall h_{1}, h_{2} & \in H e k_{1}, k_{2} \in K .
\end{aligned}
$$

O próximo teorema caracteriza as constelações de sinais que são casadas a um grupo não-comutativo.

Teorema 1 Sejam $H$ e K grupos isomorfos a $\mathbb{Z}_{n} e \mathbb{Z}_{2 m}$, respectivamente, e denotados por $H=\langle h\rangle \simeq \mathbb{Z}_{n}$ e $K=\langle k\rangle \simeq$ $\mathbb{Z}_{2 m}$, onde $\langle x\rangle$ denota o gerador do grupo $X$. Entäo, uma constelação de sinais $S$ é casada a um grupo $G=H \times{ }_{\theta} K$ se, e somente se, $S=S_{1} \times_{\varphi} S_{2}$, para $\varphi\left(\mu_{2}(k)\right)=\mu_{1} \theta(k) \mu_{1}^{-1}$, onde $\mu_{1}: H \longrightarrow S_{1}$, e $\mu_{2}: K \longrightarrow S_{2}$ são rotulamentos casados, $\theta(k) \in \operatorname{Aut}(H), \forall k \in K$ e $\theta(k)(h)=h^{-1}, \forall h \in$ $H$.

Prova: Suponha que $S$ seja uma constelação de sinais casada a $G$. Seja $\mu$ o rotulamento casado de $G$ sobre $S$. Então $\mu$ induz uma estrutura de grupo em $S$. Definindo

$$
S_{1} \triangleq \mu\left(H \times{ }_{\theta}\{e\}\right) \text { e } S_{2} \triangleq \mu\left(\{e\} \times_{\theta} K\right)
$$

temos que $S_{1}$ e $S_{2}$ são subgrupos de $S$, tal que $S_{1}$ é normal em $S$. Agora, defina $\mu_{1}: H \longrightarrow S_{1}$ e $\mu_{2}: K \longrightarrow S_{2}$ por

$$
\mu_{1}(h) \triangleq \mu(h, e) \text { e } \mu_{2}(k) \triangleq \mu(e, k) .
$$

Então é claro que $\mu_{1}, \mu_{2}$ são isomorfismos e $S=S_{1} \times_{\varphi} S_{2}$, onde $\varphi\left(\mu_{2}(k)\right)=\mu_{1} \theta(k) \mu_{1}^{-1}, \forall k \in K$. Além disso, dados $h_{1}, h_{2} \in H$,

$$
d\left(\mu_{1}\left(h_{1}\right), \mu_{1}\left(h_{2}\right)\right)=d\left(\mu_{1}(e), \mu_{1}\left(h_{1}^{-1} h_{2}\right)\right) .
$$

De modo análogo, podemos mostrar que $\mu_{2}$ é um rotulamento casado.

Reciprocamente, sejam $\mu_{1}: H \longrightarrow S_{1}$ e $\mu_{2}: K \longrightarrow S_{2}$ rotulamentos casados. Então é claro que $\mu_{1}$ e $\mu_{2}$ induzem estruturas de grupos em $S_{1}$ e $S_{2}$, respectivamente. Assim, $S=$ $S_{1} \times_{\varphi} S_{2}$ é um grupo, onde $\varphi\left(s_{2}\right) \in \operatorname{Aut}\left(S_{1}\right), \forall s_{2} \in S_{2}$, 
$\operatorname{com} \varphi\left(s_{2}\right)\left(s_{1}\right)=s_{1}^{-1}, \forall s_{1} \in S_{1}$. Uma vez que $\mu_{1}^{-1} \tau \mu_{1} \epsilon$ Aut $(H)$, para todo $\tau \in \operatorname{Aut}\left(S_{1}\right)$ segue que $G=H \times_{\theta} K$ é um grupo com $\theta(k)=\mu_{1}^{-1} \varphi\left(\mu_{2}(k)\right) \mu_{1}, \forall k \in K$. Defina $\mu: G \longrightarrow S$ como

$$
\mu(h, k) \triangleq\left(\mu_{1}(h), \mu_{2}(k)\right) .
$$

Então é fácil verificar que $\mu$ é um isomorfismo de $G$ sobre $S$. Além disso, dados $\left(h_{1}, k_{1}\right),\left(h_{2}, k_{2}\right) \in G$, temos que

$$
\begin{array}{r}
d\left(\mu\left(h_{1}, k_{1}\right), \mu\left(h_{2}, k_{2}\right)\right)= \\
d\left(\left(\mu_{1}\left(h_{1}\right), \mu_{2}\left(k_{1}\right)\right),\left(\mu_{1}\left(h_{2}\right), \mu_{2}\left(k_{2}\right)\right)\right)= \\
d\left(\mu_{1}\left(h_{1}\right), \mu_{1}\left(h_{2}\right)\right)+d\left(\mu_{2}\left(k_{1}\right), \mu_{2}\left(k_{2}\right)\right)= \\
d\left(\mu_{1}(e), \mu_{1}\left(h_{1}^{-1} h_{2}\right)\right)+d\left(\mu_{2}(e), \mu_{2}\left(k_{1}^{-1} k_{2}\right)\right),
\end{array}
$$

por outro lado,

$$
\begin{array}{r}
d\left(\mu(e, e), \mu\left(\left(h_{1}, k_{1}\right)^{-1}\left(h_{2}, k_{2}\right)\right)=\right. \\
d\left(\left(\mu_{1}(e), \mu_{2}(e)\right),\left(\mu_{1}\left(h_{1}\right), \mu_{2}\left(k_{1}\right)\right)^{-1}\left(\mu_{1}\left(h_{2}\right), \mu_{2}\left(k_{2}\right)\right)\right)= \\
d\left(\left(\mu_{1}(e), \mu_{2}(e)\right),\left(\varphi\left(\mu_{2}\left(k_{1}^{-1}\right)\right) \mu_{1}\left(h_{1}^{-1} h_{2}\right), \mu_{2}\left(k_{1}^{-1} k_{2}\right)\right)\right)= \\
d\left(\left(\mu_{1}(e), \mu_{2}(e)\right),\left(\mu_{1}\left(\theta\left(k_{1}^{-1}\right)\right)\left(h_{1}^{-1} h_{2}\right), \mu_{2}\left(k_{1}^{-1} k_{2}\right)\right)\right)= \\
d\left(\mu_{1}(e), \mu_{1}\left(h_{1} h_{2}^{-1}\right)\right)+d\left(\mu_{2}(e), \mu_{2}\left(k_{1}^{-1} k_{2}\right)\right) .
\end{array}
$$

Das expressões acima vemos que

$d\left(\mu\left(h_{1}, k_{1}\right), \mu\left(h_{2}, k_{2}\right)\right)=d\left(\mu(e, e), \mu\left(\left(h_{1}, k_{1}\right)^{-1}\left(h_{2}, k_{2}\right)\right)\right)$,

pois $d\left(\mu_{1}(e), \mu_{1}\left(h_{1}^{-1} h_{2}\right)\right)=d\left(\mu_{1}(e), \mu_{1}\left(h_{1} h_{2}^{-1}\right)\right)$. Portanto, $\mu$ é um rotulamento casado.

Corolário 2 Sejam $H$ um grupo comutativo e $K=\langle k\rangle \simeq$ $\mathbb{Z}_{2 m}$. Então, uma constelação de sinais $S$ é casada a um grupo $G=H \times_{\theta} K$ se, e somente se, $S=S_{1} \times_{\varphi} S_{2}$, para $\varphi\left(\mu_{2}(k)\right)=\mu_{1} \theta(k) \mu_{1}^{-1}$, onde $\mu_{1}: H \longrightarrow S_{1}$, e $\mu_{2}: K \longrightarrow$ $S_{2}$ são rotulamentos casados, $\theta(k) \in A u t(H), \forall k \in K e$ $\theta(k)(h)=h^{-1}, \forall h \in H$.

Note que, quando $\theta(k)=I, \forall k \in K$, com $I$ o elemento identidade do grupo $A u t(H)$, o produto semidireto $H \times_{\theta} K$ reduz-se ao produto direto. Portanto, o Teorema 1 pode ser usado para caracterizar constelações de sinais casadas a grupos comutativos.

Exemplo 3 Sejam $H=\mathbb{Z}_{3}=\{0,1,2\}, K=\mathbb{Z}_{2}=\{0,1\}$ e o homomorfismo $\theta: K \longrightarrow$ Aut $(H)$ definido por $\theta(k)=$ $\tau^{k}, k=0,1$, onde $\tau(h)=-h,: h=0,1,2$. Então $G=H \times_{\theta} K$ é um grupo não comutativo de ordem 6 com $a$ operação

$$
\begin{aligned}
(h, k)+{ }_{\theta}\left(h^{\prime}, k^{\prime}\right) & =\left(h+{ }_{3} \theta(k)\left(h^{\prime}\right), k+{ }_{2} k^{\prime}\right), \\
\forall h, h^{\prime} & \in H \text { e } k, k^{\prime} \in K,
\end{aligned}
$$

onde $+_{3} e+2$ denotam soma mod 3 e soma mod 2 , respectivamente. Assim, $G$ é isomorfo ao grupo diedral $D_{3}$. Seja $A=\{0,1,2,3,4,5\}$ um conjunto de rótulos para $G$. A tabela de Cayley de A é mostrada na Tabela 1 . Portanto, pelo Teorema 1, a constelação de sinais

$$
\begin{aligned}
S= & \left\{(1,0,1),\left(-\frac{1}{2}, \frac{\sqrt{3}}{2}, 1\right),\left(-\frac{1}{2},-\frac{\sqrt{3}}{2}, 1\right),(1,0,-1),\right. \\
& \left.\left(-\frac{1}{2}, \frac{\sqrt{3}}{2},-1\right),\left(-\frac{1}{2},-\frac{\sqrt{3}}{2},-1\right)\right\}
\end{aligned}
$$

\begin{tabular}{|c|cccccc|c|}
\hline$+_{\theta}$ & 0 & 1 & 2 & 3 & 4 & 5 & $G$ \\
\hline 0 & 0 & 1 & 2 & 3 & 4 & 5 & $(0,0)$ \\
1 & 1 & 2 & 0 & 4 & 5 & 3 & $(1,0)$ \\
2 & 2 & 0 & 1 & 5 & 3 & 4 & $(2,0)$ \\
3 & 3 & 5 & 4 & 0 & 2 & 1 & $(0,1)$ \\
4 & 4 & 3 & 5 & 1 & 0 & 2 & $(1,1)$ \\
5 & 5 & 4 & 3 & 2 & 1 & 0 & $(2,1)$ \\
\hline
\end{tabular}

Tabela 1. Tabela Cayley do grupo $\mathbb{Z}_{3} \times_{\theta} \mathbb{Z}_{2} \simeq D_{3}$.

\begin{tabular}{|c|cccccccc|c|}
\hline$+\theta$ & 0 & 1 & 2 & 3 & 4 & 5 & 6 & 7 & $G$ \\
\hline 0 & 0 & 1 & 2 & 3 & 4 & 5 & 6 & 7 & $(0,0)$ \\
1 & 1 & 2 & 3 & 0 & 5 & 6 & 7 & 4 & $(1,0)$ \\
2 & 2 & 3 & 0 & 1 & 6 & 7 & 4 & 5 & $(2,0)$ \\
3 & 3 & 0 & 1 & 2 & 7 & 4 & 5 & 6 & $(3,0)$ \\
4 & 4 & 7 & 6 & 5 & 0 & 3 & 2 & 1 & $(0,1)$ \\
5 & 5 & 4 & 7 & 6 & 1 & 0 & 3 & 2 & $(1,1)$ \\
6 & 6 & 5 & 4 & 7 & 2 & 1 & 0 & 3 & $(2,1)$ \\
7 & 7 & 6 & 5 & 4 & 3 & 2 & 1 & 0 & $(3,1)$ \\
\hline
\end{tabular}

Tabela 2. Tabela Cayley do grupo $\mathbb{Z}_{4} \times_{\theta} \mathbb{Z}_{2} \simeq D_{4}$.

ou a constelação de sinais normalizada $\tilde{S}=\frac{1}{\sqrt{2}} S$ é casada ao grupo $D_{3}$. O conjunto $S$ formado pelas 6 triplas $(a, b, c)$ foi obtido através do algoritmo apresentado na Seção 5.

Exemplo 4 Sejam $H=\mathbb{Z}_{4}=\{0,1,2,3\}, K=\mathbb{Z}_{2}=$ $\{0,1\}$ e o homomorfismo $\theta: K \longrightarrow$ Aut $(H)$ definido por $\theta(k)=\tau^{k}, k=0,1$, onde $\tau(h)=-h, h=0,1,2,3$. Então $G=H \times_{\theta} K$ é um grupo não comutativo de ordem 8 com a operação

$$
\begin{gathered}
(h, k)+_{\theta}\left(h^{\prime}, k^{\prime}\right)=\left(h+{ }_{4} \theta(k)\left(h^{\prime}\right), k+{ }_{2} k^{\prime}\right), \\
\forall h, h^{\prime} \in H \text { e } k, k^{\prime} \in K .
\end{gathered}
$$

onde $+_{4} e+{ }_{2}$ denotam soma mod 4 e soma mod 2 , respectivamente. Seja $A=\{0,1,2,3,4,5,6,7\}$ um conjunto de rótulos para $G$. A tabela de Cayley de A é mostrada na Tabela 2 , da qual podemos concluir que $G=\langle 1,4\rangle$, onde 1 e 4 denotam os geradores de $G$, é isomorfo ao grupo diedral $D_{4}$. Portanto, pelo Teorema 1, a constelação de sinais

$$
\begin{aligned}
S= & \{(1,0,1),(0,1,1),(-1,0,1),(0,-1,1) \\
& (1,0,-1),(0,1,-1),(-1,0,-1),(0,-1,-1)\}
\end{aligned}
$$

ou a constelação de sinais normalizada $\tilde{S}=\frac{1}{\sqrt{2}} S$ é casada ao grupo $D_{4}$. $O$ conjunto $S$ formado pelas 8 triplas $(a, b, c)$ foi obtido através do algoritmo apresentado na Seção 5.

\section{D-CAMINHO}

Nesta seção apresentamos uma construção de constelações de sinais a partir de um grupo qualquer via o conceito de $d$-caminho em uma $d$-cadeia baseado na construção proposta em [4].

Seja $S=\left\{\mathbf{x}_{g_{i}}: 0 \leq i \leq N-1\right\} \subset \mathbb{R}^{N}$, onde $\mathbf{x}_{g_{i}}=$ $\left(x_{\sigma_{g_{i}}(0)}, \ldots, x_{\sigma_{g_{i}}(N-1)}\right) \in \mathbb{R}^{N}$, com $g_{i} \in G, g_{0}=e \mathrm{e} \sigma_{g_{i}}$, denota a permutação associada a $g_{i}, 0 \leq i \leq N-1$. Suponha que todos os vetores distantes $d$ de $\mathbf{x}_{g_{0}}$ são $\mathbf{x}_{g_{1}}, \ldots, \mathbf{x}_{g_{r}}$. Vamos construir uma tabela dos elementos do grupo associados 


\section{Antonio de Andrade e Silva, Reginaldo Palazzo Jr Constelações de Sinais Casadas a Grupos Não-Comutativos}

com os vetores $\mathrm{x}_{g_{i}}, 0 \leq i \leq r$, da seguinte forma: a primeira linha da tabela será $g_{0}, \ldots, g_{r}$. O primeiro elemento a ser localizado na primeira coluna da tabela na $(k+1)$-ésima linha será o primeiro elemento na $k$-ésima linha que ainda não apareceu na primeira coluna da tabela. Seja $g$ o primeiro elemento na k-ésima linha que ainda não apareceu na primeira coluna da tabela. Então a $(k+1)$-ésima linha será $g, g g_{1}, \ldots, g g_{r}$. Portanto, temos a seguinte tabela:

\begin{tabular}{c|cccc} 
& $g_{0}$ & $g_{1}$ & $\ldots$ & $g_{r}$ \\
\hline$g_{0}$ & $g_{0}$ & $g_{1}$ & $\cdots$ & $g_{r}$ \\
$g_{1}$ & $g_{1}$ & $g_{1}^{2}$ & $\cdots$ & $g_{1} g_{r}$ \\
$\vdots$ & $\vdots$ & $\vdots$ & $\ddots$ & $\vdots$ \\
$g$ & $g$ & $g g_{1}$ & $\ldots$ & $g g_{r}$ \\
$\vdots$ & $\vdots$ & $\vdots$ & $\ddots$ & $\vdots$
\end{tabular}

Quando a $j$-ésima linha tiver sido escrita e todo elemento nessa $j$-ésima linha tenha aparecido uma vez na primeira coluna da tabela, o processo é interrompido e a tabela é considerada completa. Note que a tabela tem no máximo $|G|$ linhas. Agora, de $d\left(\mathbf{x}_{g_{i}}, \mathbf{x}_{g_{0}}\right)$ temos que $d\left(\mathbf{x}_{g_{i}}, \mathbf{x}_{g_{0}}\right)=$ $d\left(\mathbf{x}_{g_{j} g_{i}}, \mathbf{x}_{g_{j}}\right), 0 \leq i, j \leq N-1$, segue-se que os vetores representados pelos elementos do grupo na $2^{a}, \ldots, r$-ésima coluna na k-ésima linha estão distantes $d$ do vetor representado pelo elemento do grupo na primeira coluna dessa linha. Assim, os elementos do grupo na primeira coluna da tabela representam vetores com a propriedade de que todo vetor na correspondente linha está à distância $d$ dele. O conjunto de vetores que podem ser alcançados de $\mathbf{x}_{g_{0}}$ dessa maneira é chamado de $d$-cadeia iniciando em $x_{g_{0}},[7]$. Agora, se a distância entre os elementos do grupo na primeira coluna também estão à distância $d$ na sequência apresentada, então temse um $d$-caminho nesta $d$-cadeia. Note que o vetor $\mathbf{x}_{g_{0}}$ está incluído nesse $d$-caminho e todos os $d$-caminhos iniciando de $\mathbf{x}_{g_{0}}$ são obtidos da tabela. Com isso, provamos o seguinte teorema.

Teorema 5 Seja $\mathrm{x}_{g_{0}}: \mathrm{x}_{g_{1}}, \ldots, \mathbf{x}_{g_{k}}$ um d-caminho em uma $d$ cadeia iniciando de $\mathrm{x}_{g_{0}}$. Então

(1) $H=\left\{g_{0}, g_{1}, \ldots, g_{k}\right\}$ é um subgrupo de G gerado pelos elementos da primeira linha da tabela.

(2) Se $H \neq G$, então para todo $g \in G-H$ existe um novo dcaminho iniciando em $\mathbf{x}_{g}$ e os elementos do grupo desse novo d-caminho formam as classes laterais à esquerda $g H$ de $H$ em $G$.

Sejam $S$ uma constelação de sinais e $P(S)=\left\{S^{\prime}: S^{\prime} C\right.$ $\left.S, S^{\prime} \neq \emptyset\right\}$ uma partição de $S$. Note que cada $S^{\prime}$ pertencente à partição $P(S)$ resulta de uma classe lateral à esquerda de $H$, isto é, $g H$ em $G$. Assim, dizemos que $S$ está casada a $G$ se existe um mapeamento $\mu$ de $G$ sobre $P(S)$ tal que

$$
d(\mu(g), \mu(h))=d\left(\mu(e), \mu\left(g^{-1} h\right)\right),
$$

para todos $g, h \in G$, tal que $g H$ e $h H$ são classes laterais à esquerda de $H$ em $G$, onde $d(.,$.$) é a distância euclidiana$ quadrática interclasses laterais.
Teorema 6 Seja $G$ um grupo e seja $H$ um subgrupo de $G$ como no Teorema 5. Se $S=\bigcup_{g \in G} S_{g}$, onde $S_{g}$ são constelações de sinais associadas às classes laterais de $H$ em $G$, então $S$ está casada a $G$.

Prova. Por construção cada $S_{g}$ tem a mesma distância euclidiana quadrática intraclasses laterais e, assim, cada $S_{g}$ tem a mesma lista ordenada de distâncias euclidianas interclasses laterais, pois todas as constelações $S_{g}$ são formadas de $d$ caminhos fechados. Portanto, $\mu: G \longrightarrow P(S)$, dado por $\mu(g)=S_{g}$, é um mapeamento casado.

Pelo Teorema 6 , observamos que a constelação de sinais $S$ casada a $G$ têm a seguinte propriedade: o perfil de distância de qualquer ponto de sinal independe do ponto de sinal a ser considerado. Portanto, $G$ tem uma medida de distância invariante por translação. Assim, um código linear $C$ projetado sobre este grupo é invariante por translação (independe da palavra-código), e mais, para um canal gaussiano a probabilidade de erro condicional independe da palavra-código.

O próximo resultado considera a forma geral de decomposição de um grupo $G$ como o produto de dois grupos sendo que um deles, digamos $H$, é um subgrupo normal em $G$. Com isso, se $G / H$ é isomorfo a um grupo $K$, então $G$ é isomorfo a $H * K$ denotado por $G \simeq H * K$, segundo uma operação apropriada *. Portanto, diz-se que $G$ é uma extensão de $H$ por $K,[1]$.

Lema 7 Seja $G$ um grupo que é uma extensão de $H$ por $K$. Então,

(1) Se $S$ é uma constelação de sinais casada a $H$, então $S$ é casada a $G$.

(2) Se $S$ é uma constelação de sinais casada a $K$, então $S$ é casada $a G$.

Prova. (1) Caso particular do Teorema 3.1.

(2) Seja $\mu: G \longrightarrow S$ definida por $\mu(g)=\mu(a H)$, se $g \in a H$, para todo $g \in G$, onde $\mu$ é o rotulamento casado de $K \simeq G / H$ sobre $S$. É claro que $\mu$ está bem definida e é sobrejetiva. Dados $g_{1}, g_{2} \in G$, existem únicos $a_{1}, a_{2} \in$ $[G / H]$ e $h_{1}, h_{2} \in H$ tais que $g_{1}=a_{1} h_{1}$ e $g_{2}=a_{2} h_{2}$. Como $g_{1}^{-1} g_{2}=h_{1}^{-1} a_{1}^{-1} a_{2} h_{2} \mathrm{e}$

$$
\begin{aligned}
& h_{1}^{-1} a_{1}^{-1} a_{2} h_{2}=a_{1}^{-1}\left(a_{1} h_{1}^{-1} a_{1}^{-1}\right) a_{2} h_{2}=a_{1}^{-1} h_{3} a_{2} h_{2}, h_{3}= \\
& a_{1} h_{1}^{-1} a_{1}^{-1} \in H, \\
& h_{3} a_{2} h_{2}=a_{2}\left(a_{2}^{-1} h_{3} a_{2} h_{2}\right)=a_{2} h_{4}, h_{4}=a_{2}^{-1} h_{3} a_{2} h_{2} \in H
\end{aligned}
$$

temos que $g_{1}^{-1} g_{2}=a_{1}^{-1} a_{2} h_{4}$, isto é, $g_{1}^{-1} g_{2} \in a_{1}^{-1} a_{2} H$. Portanto.

$$
\begin{aligned}
& d\left(\mu\left(g_{1}\right), \mu\left(g_{2}\right)\right)=d\left(\mu\left(a_{1} H\right), \mu\left(a_{2} H\right)\right)= \\
& d\left(\mu(H), \mu\left(a_{1}^{-1} a_{2} H\right)\right)=d\left(\mu(e), \mu\left(g_{1}^{-1} g_{2}\right)\right) .
\end{aligned}
$$

Logo, $\mu$ é um mapeamento casado.

Note que para o mapeamento casado do Lema $7, \mu^{-1}$, não é um rotulamento casado, pois $\mu$ não é injetivo. 


\begin{tabular}{|c|cccccc|}
\hline+ & 0 & 1 & 2 & 3 & 4 & 5 \\
\hline 0 & 0 & 1 & 2 & 3 & 4 & 5 \\
1 & 1 & 2 & 0 & 4 & 5 & 3 \\
2 & 2 & 0 & 1 & 5 & 3 & 4 \\
3 & 3 & 5 & 4 & 0 & 2 & 1 \\
4 & 4 & 3 & 5 & 1 & 0 & 2 \\
5 & 5 & 4 & 3 & 2 & 1 & 0 \\
\hline
\end{tabular}

Tabela 3. Tabela de Cayley do grupo $\mathbb{D}_{3}$.

\section{ALGORITMO DE CONSTRUÇÃO DE CONSTELAÇÕES DE SINAIS CASADAS A GRUPOS}

Em geral, o Teorema 6 não fornece a natureza geométrica da constelação de sinais. De fato, este teorema estabelece que uma constelação de sinais deve ser encontrada como uma união de constelações de sinais. Assim, a chave para transformar o Teorema $6 \mathrm{em}$ um algoritmo construtivo é proporcionado pelo conceito de $d$-caminho em uma $d$-cadeia, do Teorema 5 e do Lema 7.

O algoritmo, apresentado a seguir, permite construir de maneira sistemática as figuras geométricas associadas a grupos gerais.

\section{Algoritmo A}

Dado um grupo finito $G$

Passo 1 - Associe a $G$ um conjunto qualquer de rótulos $A$;

Passo 2 - Construa a tabela de Cayley para $A$;

Passo 3 - Para cada linha da tabela de Cayley do Passo 2 associe uma permutação $\sigma_{i}, i \in A$.

Passo 4 - Selecione subconjuntos de $A$ cujos elementos do grupo associados aos vetores $\mathbf{x}_{j}$ estão à mesma distância euclidiana quadrática $d_{j}$ de $\mathbf{x}_{0}$;

Passo 5 - Para cada subconjunto $A_{j}$ de $A$ do Passo 4 associe um $d_{j}$-caminho;

Passo 6 - Associe a cada $d_{j}$-caminho do Passo 5 um subgrupo $H_{j}$ de $G$;

Passo 7 - Faça $S_{j}=\bigcup_{i \in \mathbb{I}} S_{j i}$, onde $S_{j i}$ são as constelações de sinais casadas com as classes laterais de $H_{j}$ em $G$.

Este algoritmo pode ser facilmente implementado, possibilitando a determinação de figuras geométricas associadas a grupos mais gerais do que aqueles apresentados nos exemplos a seguir.

Exemplo 8 Encontre as constelações de sinais casadas ao grupo diedral $D_{3}$.

Passo 1-Seja $A=\{0,1,2,3,4,5\}$ um conjunto de rótulos para $D_{3}$.

Passo 2-Ver Tabela 3.

$$
\text { Passo 3-Sejam }
$$

$$
\begin{gathered}
\sigma_{0}=(0), \sigma_{1}=(012)(345), \sigma_{2}=(021)(354) \\
\sigma_{3}=(03)(15)(24), \sigma_{4}=(04)(13)(25) \\
e \sigma_{5}=(05)(14)(23)
\end{gathered}
$$

as permutações associadas ao Passo 2.

\begin{tabular}{l|llll} 
& 0 & 1 & 2 & 5 \\
\hline 0 & 0 & 1 & 2 & 5 \\
1 & 1 & 2 & 0 & 3 \\
2 & 2 & 0 & 1 & 4 \\
4 & 4 & 3 & 5 & 2 \\
3 & 3 & 5 & 4 & 1 \\
5 & 5 & 4 & 3 & 0
\end{tabular}

Tabela 4. Constelação de sinais $S_{1}$, prisma.

\begin{tabular}{l|lllll} 
& 0 & 1 & 2 & 3 & 4 \\
\hline 0 & 0 & 1 & 2 & 3 & 4 \\
1 & 1 & 2 & 0 & 4 & 5 \\
2 & 2 & 0 & 1 & 5 & 3 \\
3 & 3 & 5 & 4 & 0 & 2 \\
5 & 5 & 4 & 3 & 2 & 1 \\
4 & 4 & 3 & 5 & 1 & 0
\end{tabular}

Tabela 5. Constelação de sinais $S_{2}$, antiprisma.

Passo 4-Sejam $A_{1}=\{0,1,2\},: A_{2}=\{0,3,4\} e$ $A_{3}=\{0,5\}$ os conjuntos com distâncias euclidianas

\begin{tabular}{|c|c|c|c|c|c|c|c|c|c|}
\hline & 0 & $I$ & 2 & & 0 & 3 & & & \\
\hline 0 & 0 & 1 & 2 & 0 & $\frac{0}{0}$ & $\frac{J}{3}$ & & 0 & $\frac{4}{4}$ \\
\hline 1 & 1 & 2 & 0 & 3 & 0 & 3 & 4 & 0 & 4 \\
\hline 2 & 2 & 0 & 1 & & 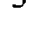 & & & & \\
\hline & 0 & 3 & 4 & & & & & & \\
\hline 0 & $\overline{0}$ & $\overline{3}$ & 4 & & & & & & \\
\hline 3 & 3 & 0 & 2 & & 0 & 5 & & & \\
\hline 2 & 2 & 5 & 3 & 0 & 0 & 5 & & & \\
\hline 5 & 5 & 2 & 1 & 5 & 5 & 0 & & & \\
\hline 1 & 1 & 4 & 5 & & & & & & \\
\hline 4 & 4 & 1 & 0 & & & & & & \\
\hline
\end{tabular}
quadráticas $d_{1}=12, d_{2}=58$ e $d_{3}=70$, respectivamente.

Passo 5-

Passo 6-Sejam $H_{1}=\{0,1,2\}, H_{2}=\{0,3\}, H_{3}=$ $\{0,4\}$ e $H_{4}=\{0,5\}$ os subgrupos de $D_{3}$ associados aos $d_{j}$-caminhos $j=1,2,3,4$ do Passo 5 .

Passo 7-Sejam $S_{1}=S_{11} \cup S_{13}$, onde $S_{11}=\{0,1,2\} e$ $S_{13}=\{0,5\}, S_{2}=S_{21} \cup S_{22}$, onde $S_{21}=\{0.1 .2\}$ e $S_{22}=\{0,3,4\}$, onde os d-caminhos associados são mostrados nas primeiras colunas nas Tabelas 4 e 5 .

Os gráficos associados a $S_{2}$ e $S_{1}$ são o antiprisma da Figura 1 e o prisma da Figura 2, respectivamente. Note das Tabelas 4 e 5 que os vizinhos de 3 são 4,5 e 1, no caso do conjunto de sinais $S_{1}$, e 5,4,0, e 2, no caso do conjunto de sinais $S_{2}$, respectivamente.Pelo Lema 7 as constelações de sinais $2 P S K$ e $3 P S K$ são também casadas com o grupo $D_{3}$.

\section{CONCLUSÕES}

Neste trabalho consideramos o problema de casamento de uma constelação de sinais a um grupo, o qual é o produto semidireto de um grupo comutativo por um grupo cíclico de 


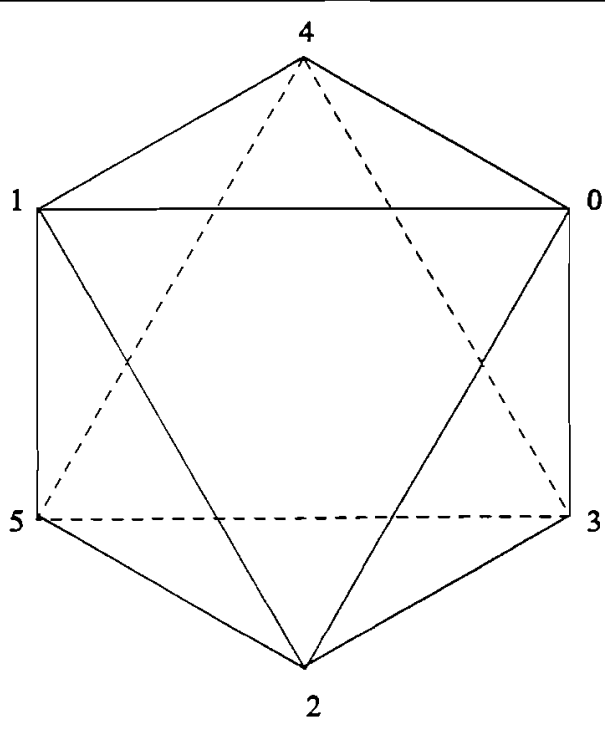

Figura 1. Antiprisma casado com $D_{3}$.

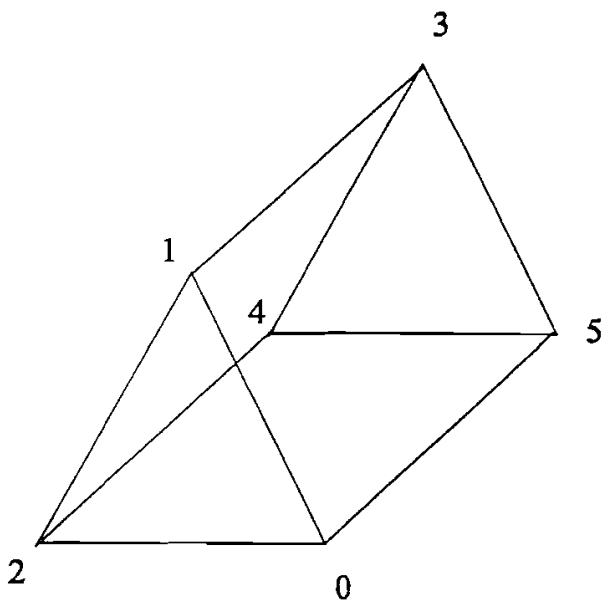

Figura 2. Prisma casado com $D_{3}$. groups," Proc. IEEE Internat. Symp. on Inform. Theory, ISTT-94, Trondhein, Norway, 1994.

[5] A.A. e Silva e R. Palazzo, Jr., "Construção de reticulados via fórmula de códigos p-ários generalizado," 13 Simpósio Brasileiro de Telecomunicações, Águas de Lindóia, São Paulo, pp. 66-70, 1995.

[6] A.A. e Silva, Uma contribuição à classe dos códigos geometricamente uniformes, Tese de Doutorado, FEECUNICAMP, 1996.

[7] D. Slepian, "Groups codes for the gaussian channel," Bell Syst. Tech. J., vol 47, pp 576-602, 1968.

Antonio de Andrade e Silva é Professor no Departamento de Matemática da Universidade Federal da Paraíba, João Pessoa. Suas áreas de interesse são Teoria da Codificação e Álgebra.

Reginaldo Palazzo Jr. graduou-se em Engenharia Elétrica e obteve o título de Mestre em Engenharia Elétrica ambos pela Faculdade de Engenharia Elétrica da UNICAMP, em 1975 e 1977, respectivamente. Em seguida obteve o graus de Engineer e de Ph.D. pela University of California, Los Angeles, CA, USA, em 1981 and 1984, respectivamente. Desde maio de 1985 pertence ao quadro de docentes da Faculdade de Engenharia Elétrica e de Computação, FEEC-UNICAMP, onde obteve a Livre-Docência em 1987. Desde 1996 é Professor Titular e Coordenador do Grupo de Codificação Algébrica e Geométrica na FEEC. Suas áreas de pesquisa são teoria da codificação, da informaçãa, de comunicações e criptografia.

ordem par. Os resultados de Loeliger foram estendidos e as condições para as quais o casamento de sinais a grupos não abelianos possa ser efetivado foram estabelecidas. Um algoritmo para encontrar as correspondentes constelações de sinais casadas a tais grupos foi proposto. A extensão do Teorema 1 para um grupo mais geral pode ser considerada como uma proposta de pesquisa.

\section{REFERÊNCIAS}

[1] D. S. Dummit and R.M. Foote, Abstract Algebra, New Jersey, Prentice Hall, 1991.

[2] G. D. Forney Jr, "Geometrically uniform codes," IEEE Trans. Inform. Theory, vol. IT-37, pp 1241-1260, Sept. 1991.

[3] H. A. Loeliger, "Signal sets matched to groups," IEEE Trans. Inform. Theory, vol. IT-37, pp 1675-1682, Nov. 1991.

[4] R. Palazzo Jr, J. C. Interlando e C. Almeida, "Constructions of signals sets matched to abelian and non-abelian 